\title{
Silymarin-mediated regulation of the cell cycle and DNA damage response exerts antitumor activity in human hepatocellular carcinoma
}

\author{
HONG CUI $^{1}$, TIE-LING LI ${ }^{2}$, HAI-FENG GUO ${ }^{3}$, JIA-LIANG WANG ${ }^{1}$, PING XUE ${ }^{4}$, \\ YING ZHANG $^{5}$, JING-HUI FAN ${ }^{1}$, ZHI-PING LI $^{1}$ and YUE-JUAN GAO ${ }^{1}$
}

\begin{abstract}
${ }^{1}$ Department of Pharmacy, The Red Flag Hospital of Mudanjiang Medical College; ${ }^{2}$ Department of Pathology, Mudanjiang Medical College; Departments of ${ }^{3}$ General Surgery and ${ }^{4}$ Respiration, The Red Flag Hospital of Mudanjiang Medical College; ${ }^{5}$ Department of Pharmacy, Mudanjiang Medical College, Mudanjiang, Heilongjiang 157011, P.R. China
\end{abstract}

Received September 8, 2016; Accepted October 26, 2017

DOI: $10.3892 / \mathrm{ol} .2017 .7425$

\begin{abstract}
A novel module-search algorithm method was used to screen for potential signatures and investigate the molecular mechanisms of inhibiting hepatocellular carcinoma (HCC) growth following treatment with silymarin (SM). The modules algorithm was used to identify the modules via three major steps: i) Seed gene selection; ii) module search by seed expansion and entropy minimization; and iii) module refinement. The statistical significance of modules was computed to select the differential modules (DMs), followed by the identification of core modules using the attract method. Pathway analysis for core modules was implemented to identify the biological functions associated with the disease. Subsequently, results were verified in an independent sample set using reverse transcription polymerase chain reaction (RT-PCR). In total, 18 seed genes and 12 DMs (modules 1-12) were identified. The core modules were isolated using gene expression data. Overall, there were 4 core modules (modules 11, 5, 6 and 12). Additionally, DNA topoisomerase 2-binding protein 1 (TOPBPI), non-structural maintenance of chromosomes condensing I complex subunit $\mathrm{H}$, nucleolar and spindle associated protein 1 (NUSAPI) and cell division cycle associated $3(C D C A 3)$ were the initial seed genes of module 11, 5, 6 and 12, respectively. Pathway results revealed that cell cycle signaling pathway was enriched by all core modules simultaneously. RT-PCR results indicated that the level of CDCA3, TOPBPI and NUSAPI in SM-treated HCC samples was markedly decreased compared with that in non-SM-treated HCC. No statistically significant difference
\end{abstract}

Correspondence to: Professor Yue-Juan Gao, Department of Pharmacy, The Red Flag Hospital of Mudanjiang Medical College, 5 Tongxiang Road, Aimin, Mudanjiang, Heilongjiang 157011, P.R. China

E-mail: gaoyuejuanedu@sina.com

Key words: hepatocellular carcinoma, silymarin, differential co-expression network, differential modules, DNA topoisomerase 2-binding protein 1 between the transcriptional levels of $C D C A 3$ in SM-treated and non-treated HCC groups was identified, although $C D C A 3$ expression was increased in the treated group compared with the untreated group. Furthermore, although the expression level of TOPBP1 and NUSAP1 in the SM-treated group was decreased compared with that in the normal group, no significant difference was observed. From the results of the present study it can be inferred that TOPBPI, NUSAP1 and $C D C A 3$ of the core modules may serve notable functions in SM-associated growth suppression of HCC.

\section{Introduction}

Currently, hepatocellular carcinoma (HCC) is the sixth most prevalent malignancy (1) and the second leading cause of cancer-associated mortality worldwide (2). Notably, $\sim 50 \%$ of new cases globally were in China in 2012 (3). Despite evidence that the utilization of chemotherapeutic drugs has improved the clinical outcome of patients with HCC $(4,5)$, the effects have been limited owing to drug resistance and toxicity. Thus, investigation of novel non-toxic therapeutic drugs with the ability to prevent $\mathrm{HCC}$ growth is urgently required.

Milk thistle extract is non-toxic and has been applied to protect against liver damage in clinical settings, as well as experimental models, for $>2,000$ years (6). Silymarin (SM), a complex mixture of flavonoids, is extracted from the seeds of the milk thistle (Silybum marianum). Notably, SM has been used as a complementary and alternative treatment for patients with cancer $(7,8)$. Nevertheless, the molecular mechanisms of anti-HCC-associated effects of SM remain poorly understood.

Network biology has been demonstrated to be a powerful tool for analyzing complex molecular networks that exert important functions in the development and progression of disease $(9,10)$. Previously, studies have applied dynamic changes in molecular networks to predict disease outcomes $(11,12)$. Since transcriptome information is the most abundant form of omics data, co-expression networks have been employed in the majority of studies (13-15). However, a limitation of these previous studies was that networks were built using only co-expression data; this decreases the statistical power to screen pathways that 
are abnormal in diseased conditions (16). Selecting groups of genes that demonstrate coherent differential activities between healthy and diseased conditions is a more powerful approach. These gene groups directly capture the affected pathways. Thus, it is useful to identify coherent differentially expressed gene modules with common members, yet varied connectivity in differential co-expression networks (DCNs) (11).

The present study utilized a novel module-search algorithm method to screen for potential signatures and to investigate the molecular mechanisms underlying anti-HCC associated effects following SM treatment. First, gene expression data from SM-treated HCC and control samples, generated by Lovelace et al (17), were downloaded from the European Molecular Biology Laboratory-European Bioinformatics Institute (EMBL-EBI) database (www.ebi.ac.uk). Subsequently, the construction of DCN was implemented if two connected genes exhibited associated expression patterns across conditions and if the expression levels of these two genes were markedly different between the SM-treated HCC and control condition. Following that, the DCN was analyzed to identify modules through three major steps: i) Seed gene selection; ii) module search by seed expansion and entropy minimization; and iii) module refinement. The statistical significance of modules was then computed to select the differential modules (DMs); core modules were detected using the attract method (18), followed by pathway enrichment analysis for core modules. Finally, validation tests were implemented to verify the results. The present study aimed to contribute to the understanding of potential action mechanisms associated with SM inhibition of HCC growth.

\section{Materials and methods}

Microarray data. The gene expression profile dataset E-GEOD-50994, generated by Lovelace et al (17), was downloaded from the EMBL-EBI database (www.ebi.ac.uk/), based on the A-AFFY-141 platform of the Affymetrix Human Gene 1.0 ST Array (HuGene-1_0-st). Gene expression data of E-GEOD-50994, containing 10 human SM-treated HCC samples and 14 human dimethyl sulfoxide-treated HCC control group samples, were obtained and the probes were mapped to the gene symbols. A total of 12,227 genes were identified.

Protein-protein interaction network (PPIN). The PPIN ensemble (the network constructed by all PPI interactions) containing 787,896 interactions and 16,730 genes was obtained from the String database (string.embl.de; accessed June, 2016). Subsequently, the 12,227 genes identified from the aforementioned microarray data were mapped to the PPIN and a novel PPIN was created.

DCN construction. DCN construction comprised two steps. First, a binary co-expression network was constructed prior to assignment of edge weight based on differential gene expression between the SM-treated-HCC and control groups. To construct the binary gene co-expression network, edges were selected according to the absolute value of the Pearson's correlation coefficient (PCC) of the expression profiles of two genes. Briefly, after obtaining gene expression values between the SM-treated-HCC and control groups, the PCC of the interactions of a novel PPIN in different conditions were computed (SM-treated HCC and control samples), determined as A1 and A2. Similarly, the absolute value of the difference of PCC between two groups, marked as $\delta$-values, was also computed. In an attempt to eliminate indirect correlation due to a third gene, the utilization of the first order partial PCC was implemented, as previously described (19). Only edges with correlations greater than the pre-defined threshold $\delta$-values were chosen. In the current study, the $\delta$-value was set at 0.9 , such that the maximal number of genes was connected in the DCN to be constructed.

Subsequently, edge weights were assigned in the binary co-expression network based on the P-value of differential gene expression in SM-treated HCC and control conditions. In the present study, a one-sided Student's t-test was applied to identify differential gene expression for microarray data. The weight $\mathrm{w}_{\mathrm{i}, \mathrm{j}}$ on edge $(\mathrm{i}, \mathrm{j})$ in the DCN was defined as follows:

$$
\mathrm{w}_{\mathrm{i}, \mathrm{j}}=\left\{\frac{\left(\log \mathrm{p}_{\mathrm{i}}+\log \mathrm{p}_{\mathrm{j}}\right)^{1 / 2}}{\left(2^{*} \max _{0} 1 \in \mathrm{V}\left|\log \mathrm{P}_{\mathrm{l}}\right|\right)^{1 / 2}}, \text { if } \operatorname{cor}(\mathrm{i}, \mathrm{j}) \geq \delta, \text { if } \operatorname{cor}(\mathrm{i}, \mathrm{j})<\delta\right. \text {. }
$$

where $p_{i}$ and $p_{j}$ were respective P-values of differential expression for gene ${ }_{i}$ and gene $_{j}$. V denoted the number of nodes in the DCN, and cor(i,j) represented the absolute value of PCC

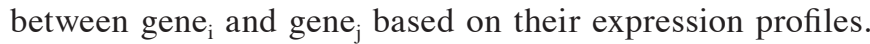
Under the weighting scheme, genes that were co-expressed and demonstrated marked differential expression were assigned higher weights.

Identification of modules in DCN. Module search is used to screen gene modules with common members but varied connectivity across multiple molecular interaction networks (11). The identification of modules comprises three stages: i) Seed prioritization; ii) module identification by seed expansion; and iii) entropy minimization and refinement of candidate modules.

Seed prioritization. Seed prioritization sorted genes in the DCN using the topological measurement (degree) analysis. In brief, for $\mathrm{DCN}, \mathrm{Gk}=\left(\mathrm{V}, \mathrm{E}_{\mathrm{k}}\right)(1 \leq \mathrm{k} \leq \mathrm{M})$ with an adjacency matrix $A_{k}=\left(a_{i j k}\right)_{n x n}$, a function was constructed to compute the importance of vertex $i$ as follows: $g(i)=\sum_{j \in n k(i)} A_{i j k}^{\prime} g(j)$, where Gk refers to DCN, $a_{i j k}$ denoted the weight on the edge $(i, j), w_{i, j}$, in network Gk, V refers to the count of nodes in the DCN Gk, $\mathrm{g}$ (i) stands for the importance of vertex $\mathrm{i}$ in the $\mathrm{DCN}, \mathrm{N}_{\mathrm{k}}$ (i) was the set of neighbors of gene i in $\mathrm{G}_{\mathrm{k}}$; $\mathrm{A}_{\mathrm{k}}$ 'represented the degree normalized weighted adjacency matrix that was calculated as $\mathrm{A}_{\mathrm{k}}^{\prime}=\mathrm{D}^{-1 / 2} \mathrm{~A}_{\mathrm{k}} \mathrm{D}^{1 / 2}$ where $\mathrm{D}$ was the diagonal matrix.

For each gene, DCN rank was acquired, marked as $g=\left[g^{(1)}, \ldots, g^{(M)}\right]$, then a $\mathrm{z}$-score was computed for each rank $g^{(1)}$. Subsequently, the rank was obtained for that gene in the DCN by averaging the $\mathrm{z}$-scores. The top $1 \%$ of genes was extracted as the seed genes.

Module search. Module search repeatedly included genes whose addition caused the maximum decrease in the graph entropy-based objective function until there was no decrease in the objective function. For a given seed gene $\mathrm{v} \varepsilon \mathrm{V}, \mathrm{V}$ was treated as a module $C=\{v\}$. For each vertex, $\mathrm{u}$, in its neighborhood in the DCN, $\mathrm{N}(\mathrm{v})$ was defined as $\mathrm{N}(\mathrm{v})=\mathrm{U}_{\mathrm{i}} \mathrm{N}_{\mathrm{i}}(\mathrm{v})$, where 
$\mathrm{N}_{\mathrm{i}}(\mathrm{v})$ was the neighbor set in $\mathrm{G}_{\mathrm{i}}$ as the candidate for module C. For each u $\varepsilon \mathrm{N}(\mathrm{v})$, the entropy decrease was computed between the novel module $\mathrm{C}^{\prime}=\mathrm{CU}\{\mathrm{v}\}$ and $\mathrm{C}$, that was to say, $\Delta \mathrm{H}\left(\mathrm{C}^{\prime}, \mathrm{C}\right)=\mathrm{H}(\mathrm{C})-\mathrm{H}\left(\mathrm{C}^{\prime}\right)$. If $\Delta \mathrm{H}\left(\mathrm{C}^{\prime}, \mathrm{C}\right)$ was $>0$; this meant the addition of vertex $\mathrm{u}$ improved the connectivity of the module $\mathrm{C}$. The vertex $\mathrm{u}$ whose addition maximizes $\Delta \mathrm{H}$ was added to module $\mathrm{C}$. If there were more than one vertex contained at each stage, one was randomly selected. The expansion stage stopped when no additional vertex could further decrease the entropy of the evolving module.

Refinement of candidate modules. In the refinement stage, the modules with node sizes $<5$ were removed. Jaccard index (11), the ratio of intersection over union for two sets, was utilized to merge the overlapping modules. In the present study, a Jaccard index not $<0.5$ was used.

Statistical significance of modules. The statistical significance of modules was computed on the basis of the null score distribution of modules produced by randomized networks. In the present study, the networks with the same size of interactions in DCN that were extracted from the novel PPIN were determined as randomized networks. Each network was completely randomized 100 times through degree-preserved edge shuffling. In an attempt to obtain the null distribution of module scores, a module search was performed on the randomized networks. Next, the empirical P-value of a module was computed as the probability of the module having a smaller score by chance based on the following formula: $\mathrm{P}=\sum$ (count $\left(\mathrm{H}_{\mathrm{R}}\right)>$ count $\left.\left(\mathrm{H}_{\mathrm{DCN}}\right)\right)$ /count $\left(\mathrm{H}_{\mathrm{R}}\right)$, Where the count $\left(\mathrm{H}_{\mathrm{R}}\right)$ was the number of modules generated by randomized networks, count $\left(\mathrm{H}_{\mathrm{DCN}}\right)$ denoted the number of modules produced by DCN.

After obtaining P-values, the false discovery rate (FDR) was used to adjust the P-values, which was computed using the Benjamini \& Hochberg method (20). In the present study, FDR $\leq 0.05$ was regarded as significant. To select activated core modules whose expression distinguished control and SM-treated HCC samples, the attract method (18) was used. Modules with a FDR $\leq 0.05$ were considered as core modules.

Pathway enrichment analysis for genes of core modules. Kyoto Encyclopedia of Genes and Genomes (KEGG) is a reference knowledge database for comprehending biological processes through pathway aligning to map genes to reference pathways to deduce cell behaviors (21). In the present study, to understand the biological functions of core modules further, the Database for Annotation, Visualization and Integrated Discovery (DAVID; https://david.ncifcrf.gov/home.jsp) (22) was utilized to perform pathway enrichment analysis for the genes in core modules to identify significant pathways. Significant pathways were extracted when the FDR was set as 0.01 .

Validation test of effects of SM on key genes

Patient subjects. In total, 40 patients with HCC and 20 healthy volunteers were enrolled in the present study. Among these 40 patients, 20 subjects were treated with SM, whereas the remaining 20 cases were not. Furthermore, 20 healthy volunteers were included as the normal control group. No significant differences were observed in sex, age, case history or regional
Table I. Sequence of primers of the selected genes.

Gene Primer sequence, 5'-3'

\section{TOPBPI}

Forward GAACTTGCCCCCTAGTGGTGAACTCTTACT

Reverse ATCACGGAAGCCACGTCCTCTCGGCGTCAA

\section{$C D C A 3$}

Forward GAATTCGGTTGAGATGGGCTCAGCCAA

Reverse TCTAGAGCCCTGGGTGACTGCATTGCT

\section{NUSAP1}

\section{Forward AAACTTACAAACAACCCCATCTCC}

Reverse GTTTCTTCGGTTGCTCTTCCTTT

\section{$\beta$-actin}

\section{Forward CTCCATCCTGGCCTCGCTGT Reverse GCTGTCACCTTCACCGTTCC}

TOPBP1, DNA topoisomerase 2-binding protein 1; $C D C A 3$, cell division cycle associated 3 ; NUSAP1, nucleolar and spindle associated protein 1.

distribution. Among these 40 patients, there were 23 females and 17 males. The average age was $48.34 \pm 5.14$ years (range, 42-56 years). All participates were recruited between May 2014 and May 2015 in The Red Flag Hospital (Mudanjiang, China). The experimental protocol was approved by the Human Research Ethics Committee and the Confidentiality of Health Information Committee of The Red Flag Hospital. Written informed consent was obtained from all participants. HCC tissues were obtained from the SM-treated and untreated HCC groups, and liver tissues was obtained from the normal control samples to conduct the subsequent experiments.

RT-PCR analysis. A TRIzol RNA extraction kit (cat. no. 15596-026; Invitrogen; Thermo Fisher Scientific, Inc., Waltham, MA, USA) to extract total RNA from the HCC tissues. Next, SuperScript II RNase H reverse transcriptase (Invitrogen; Thermo Fisher Scientific, Inc.) was used to synthesize cDNA. The cDNAs were sub-packaged and stored at $-20^{\circ} \mathrm{C}$. Subsequently, synthesized cDNAs were utilized as templates for PCR amplification and $\beta$-actin was used as an internal control gene to check the consistency of RT. The sequences of primers of selected genes for PCR amplification are listed in Table I. PCR amplification was conducted by means of the following experimental protocol: $10 \mu \mathrm{l}$ 10X PCR buffer (Eurogentec Ltd., Seraign, Belgium), $1 \mu$ l TaqDNA polymerase (Eurogentec Ltd.; $5 \mathrm{U} / \mu \mathrm{l}), 3 \mu \mathrm{l}$ upstream primers, $3 \mu \mathrm{l}$ downstream primers and $8 \mu \mathrm{l}$ dNTPs. Table II presented the amplification thermocycling conditions. PCR products were run on a $1.5 \%$ agarose gel at $100 \mathrm{mV}$ for $30 \mathrm{~min}$, stained with ethidium bromide $(5 \mu \mathrm{l} / 100 \mathrm{ml})$, visualized using a UV transilluminator, and analyzed using Quantity One software (v.4.2, Bio-Rad Laboratories, Hercules, CA, USA). The experiment was repeated three times and plotted as the mean \pm standard deviation.

Statistical analysis. Feature Extraction software (version 10.7; Agilent Technologies, Inc., Santa Clara, CA, USA) was used 
Table II. Polymerase chain reaction amplification conditions.

\begin{tabular}{|c|c|}
\hline Gene & Response conditions \\
\hline ТОРВР1 & $\begin{array}{l}\text { Pre-degeneration at } 95^{\circ} \mathrm{C} \text { for } 2 \mathrm{~min} \text {, degeneration at } 94^{\circ} \mathrm{C} \text { for } 30 \mathrm{sec} \text {, } \\
\text { annealing at } 58^{\circ} \mathrm{C} \text { for } 30 \mathrm{sec} \text {, extension at } 72^{\circ} \mathrm{C} \text { for } 30 \mathrm{sec} \text { for } \\
40 \text { cycles and extension at } 72^{\circ} \mathrm{C} \text { for } 10 \mathrm{~min} \text {. }\end{array}$ \\
\hline$C D C A 3$ & $\begin{array}{l}\text { Pre-degeneration at } 95^{\circ} \mathrm{C} \text { for } 2 \mathrm{~min} \text {, degeneration at } 94^{\circ} \mathrm{C} \text { for } 1 \mathrm{~min} \text {, } \\
\text { annealing at } 62^{\circ} \mathrm{C} \text { for } 30 \mathrm{sec} \text {, extension at } 68^{\circ} \mathrm{C} \text { for } 1 \mathrm{~min} \text { for } \\
45 \text { cycles and extension at } 72^{\circ} \mathrm{C} \text { for } 7 \mathrm{~min} \text {. }\end{array}$ \\
\hline NUSAP1 & $\begin{array}{l}\text { Pre-degeneration at } 95^{\circ} \mathrm{C} \text { for } 5 \mathrm{~min} \text {, degeneration at } 95^{\circ} \mathrm{C} \text { for } 20 \mathrm{sec} \text {, } \\
\text { annealing at } 56^{\circ} \mathrm{C} \text { for } 30 \mathrm{sec} \text {, extension at } 72^{\circ} \mathrm{C} \text { for } 30 \mathrm{sec} \text { for } \\
40 \text { cycles and extension at } 72^{\circ} \mathrm{C} \text { for } 7 \mathrm{~min} \text {. }\end{array}$ \\
\hline$\beta$-actin & $\begin{array}{l}\text { Pre-degeneration at } 94^{\circ} \mathrm{C} \text { for } 3 \mathrm{~min} \text {, degeneration at } 94^{\circ} \mathrm{C} \text { for } 60 \mathrm{sec} \text {, } \\
\text { annealing at } 56^{\circ} \mathrm{C} \text { for } 30 \mathrm{sec} \text {, extension at } 72^{\circ} \mathrm{C} \text { for } 60 \mathrm{sec} \text { for } \\
40 \text { cycles and extension at } 72^{\circ} \mathrm{C} \text { for } 7 \mathrm{~min} \text {. }\end{array}$ \\
\hline
\end{tabular}

TOPBP1, DNA topoisomerase 2-binding protein 1; CDCA3, cell division cycle associated $3 ; N U S A P 1$, nucleolar and spindle associated protein 1.

to analyze the statistical significance of the microarray results. The original data were normalized based on the Quantile Normalization Algorithm (Agilent Technologies, Inc.). Quantile normalization was conducted using function 'normalize. quantiles' from an R package (23). PCC was used to calculate the edge weight. One-sided Student's t-test was applied to identify differential gene expression for microarray data between two groups. One-way analysis of variance, followed by Student-Newman-Keuls post hoc test, was used to assess the changes of the key genes in verification test across more than two conditions. The FDR was calculated to correct the raw P-values. The threshold value applied to designate core module was FDR $\leq 0.05$, and the cut-off criteria for significant pathways was FDR $<0.01$. $\mathrm{P}<0.05$ was considered to indicate a statistically significant difference. In the present study, SPSS version 18.0 (SPSS, Inc., Chicago, IL, USA) was used for statistical analysis.

\section{Results}

Overview of the module-search algorithmfor screening shared co-expression modules in DCN. In an attempt to measure the dynamics of pathway connectivity, a novel method, module-search algorithm, was utilized to identify shared sub-networks in the DCN. These sub-networks were modules that comprised the same set of genes yet potentially different connectivity among the gene members. By means of this algorithm, the dynamic changes in module connectivity were quantified. Coupled with network topological measurements, the prior probabilities were utilized to sort and select seed genes. Module identification problems were transformed into minimum entropy problems by adding a graph-entropy-based objective function to the modules. Empirical P-values of candidate modules were determined through randomized networks.

Construction of DCN. The genes from the microarray data were aligned to the ensemble PPIN, a novel PPIN including 10,859 genes and 351,047 interactions was obtained. To construct a gene co-expression network, edges were selected
Table III. Seed genes and the distribution of z-scores.

\begin{tabular}{rlr}
\hline Row & \multicolumn{1}{c}{ Gene } & Z-score \\
\hline 1 & GMNN & 329.58 \\
2 & NCAPG & 290.38 \\
3 & KIF11 & 284.85 \\
4 & CCNB1 & 272.13 \\
5 & NCAPH & 234.93 \\
6 & NUSAP1 & 227.92 \\
7 & DLGAP5 & 226.55 \\
8 & ECT2 & 214.57 \\
9 & NUP107 & 208.83 \\
10 & NDC80 & 201.93 \\
11 & ANLN & 201.44 \\
12 & KIF20A & 189.95 \\
13 & TOPBP1 & 187.53 \\
14 & GINS1 & 182.10 \\
15 & CDCA3 & 180.81 \\
16 & ARHGAP11A & 169.74 \\
17 & ASPM & 167.06 \\
18 & KIF2C & 166.63 \\
& & \\
& &
\end{tabular}

according to the absolute value of PCC of the expression profiles of two genes. With $\delta>0.9$, a total of 3,395 edges and 1,876 nodes were selected to construct the DCN.

Identification of modules in DCN. On the basis of the z-score distribution of 1,876 nodes in DCN, 18 genes possessed a top $1 \% \mathrm{z}$-score value and were selectively termed as seed genes (Table III). Among these 18 seed genes, the z-score values of 4 genes were $>270$, for example: Geminin, DNA replication inhibitor (z-score, 329.58); non-SMC condensin I complex, subunit G (z-score, 290.38); kinesin family member 11 (z-score, 284.85); and cyclin B1 (z-score, 272.13). Subsequently, a 
Table IV. Core modules and initial seed genes.

\begin{tabular}{lll}
\hline Module & FDR & Initial seed genes \\
\hline 11 & $6.96 \times 10^{-5}$ & TOPBP1 \\
5 & $1.01 \times 10^{-3}$ & NCAPH \\
6 & $1.13 \times 10^{-2}$ & NUSAP1 \\
12 & $1.13 \times 10^{-2}$ & CDCA3 \\
\hline
\end{tabular}

TOPBP1, DNA topoisomerase 2-binding protein 1; CDCA3, cell division cycle associated $3 ; N U S A P 1$, nucleolar and spindle associated protein $1 ; N C A P H$, non-SMC condensing I complex, subunit $\mathrm{H}$; FDR, false discovery rate.

module search was performed by taking the 18 seed genes as initial genes, according to entropy decrease, to identify candidate modules. Following the removal of modules with $<5$ nodes, the modules were merged based on a Jaccard index $\geq 0.5$, and 12 modules (modules 1-12) were identified.

Identification of DMs. On the basis of the FDR distribution, it was revealed that the FDR values of the aforementioned 12 modules were $<0.05$, indicating significance. Using gene expression data from SM-treated-HCC and control samples, the core modules whose differential expression distinguished between the treated and control samples were isolated using the attract method. On the basis of the criteria, 4 core modules were observed including

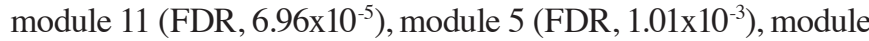

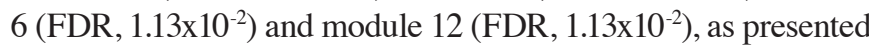
in Table IV. Among these, there were 73 nodes and 420 edges in module 11 (Fig. 1). Furthermore, out of the 73 nodes, 16 genes were seed genes and the initial gene was DNA topoisomerase 2-binding protein 1 (TOPBPI). As presented in Fig. 2, there were 74 nodes and 553 edges in module 5, involving 18 seed genes. Notably, the initial seed gene was non-SMC condensing I complex, subunit $\mathrm{H}(\mathrm{NCAPH})$. Fig. 3 demonstrates that there were 86 nodes and 634 edges in module 6 , which was also involved in 18 seed genes. In addition, nucleolar and spindle-associated protein 1 (NUSAPI) was the initial seed gene. As presented in Fig. 4, module 12 comprised 46 nodes and 315 edges; furthermore, there were 16 seed genes and cell division cycle associated 3 (CDCA3) was the initial seed gene.

Pathway enrichment analysis for genes in the core modules. Using DAVID and KEGG, pathway enrichment analysis of genes in the core modules was conducted. When the threshold criteria of FDR $<0.05$ was applied, two pathways for module 12 were identified (cell cycle and progesterone-mediated oocyte maturation). Module 11 was primarily enriched in two biological process terms; cell cycle and RNA transport. Module 5 was only involved in one pathway (cell cycle). Module 6 was involved in two pathways, including cell cycle and RNA transport. Notably, the cell cycle pathway was simultaneously enriched by all core modules. Specific information is presented in Table V.

Validation test. In the present study, PCR was employed to verify the mRNA expression levels of key genes (TOPBP1, $C D C A 3$ and NUSAPI) identified from bioinformatic analysis.
The relative expression level of these genes examined by PCR is presented in Fig. 5. The expression level of TOPBP1, CDCA3 and NUSAPI were significantly increased in the non-treated HCC group compared with that in normal group $(\mathrm{P}<0.05)$. Furthermore, following SM treatment in HCC samples, the expression levels of $C D C A 3(\mathrm{P}<0.05)$, TOPBPI $(\mathrm{P}<0.001)$ and NUSAP1 $(\mathrm{P}<0.001)$ were significantly decreased compared with untreated HCC. Furthermore, although the expression level of TOPBP1 and NUSAPI in SM-treated HCC group was decreased compared to that in normal group, no statistical difference was observed $(\mathrm{P}>0.05)$. In addition, there was no difference in the expression level of $C D C A 3$ between the normal and SM-treated HCC group $(\mathrm{P}>0.05)$, despite $C D C A 3$ being increased in the SM-treated HCC group compared with that in normal.

\section{Discussion}

The utilization of SM has previously been recommended for the treatment of liver conditions, including toxin-induced liver damage, and as a supportive treatment for chronic inflammatory liver conditions (24). Nevertheless, the underlying molecular mechanisms of SM on HCC remain not fully understood. In the present study, novel gene modules for investigating the mechanisms of anti-HCC effects of SM were analyzed using the module-search algorithm. The results of the present study demonstrated that a total of $12 \mathrm{DMs}$ were selected. Among these DMs, modules 11, 5, 6 and 12 were core modules with initial seed genes TOPBP1, NCAPH, NUSAP1 and $C D C A 3$, respectively. Notably, it was revealed that the cell cycle pathway was simultaneously enriched by all core modules. Furthermore, the expression verification of TOPBP1, NUSAP1 and CDCA3 using RT-PCR identified that SM was able to inhibit the progression of HCC by decreasing the expression of these key genes.

TOPBP1, which interacts with topoisomerase II $\beta$, has been demonstrated to be necessary for mitotic progression (25). Evidence has demonstrated that TOPBP1 co-localizes with breast cancer type 1 susceptibility protein at dysregulated replication forks and exerts important functions in the DNA damage response and DNA replication (26). Generally, DNA damage response disrupts the cell cycle by triggering checkpoint mechanisms; the inability to control a normal DNA damage response results in genomic instability is a hallmark of the majority of different types of cancer $(27,28)$. Notably, a previous study demonstrated that SM is able to decrease DNA damage in skin cancer (29). In light of these aforementioned studies, it may be inferred that the inhibitory effect of SM on HCC development, by regulating TOPBP1, highlights the potential use of SM in controlling the DNA damage response in HCC.

NUSAP1, a significant mitotic regulator, is crucial for numerous cellular events during mitosis including spindle assembly and cytokinesis (30). Furthermore, the deregulation of mitosis is a common characterization of the majority of different types of cancer $(31,32)$. In previous studies, NUSAPl has been demonstrated to be upregulated in several malignant types of cancer, including breast cancer (33) and HCC (34). Notably, deletion of NUSAPI has been revealed to arrest the cell cycle in $\mathrm{G}_{2}-\mathrm{M}$ phase (30). A previous study demonstrated that $\mathrm{SM}$ treatment markedly reduced the 


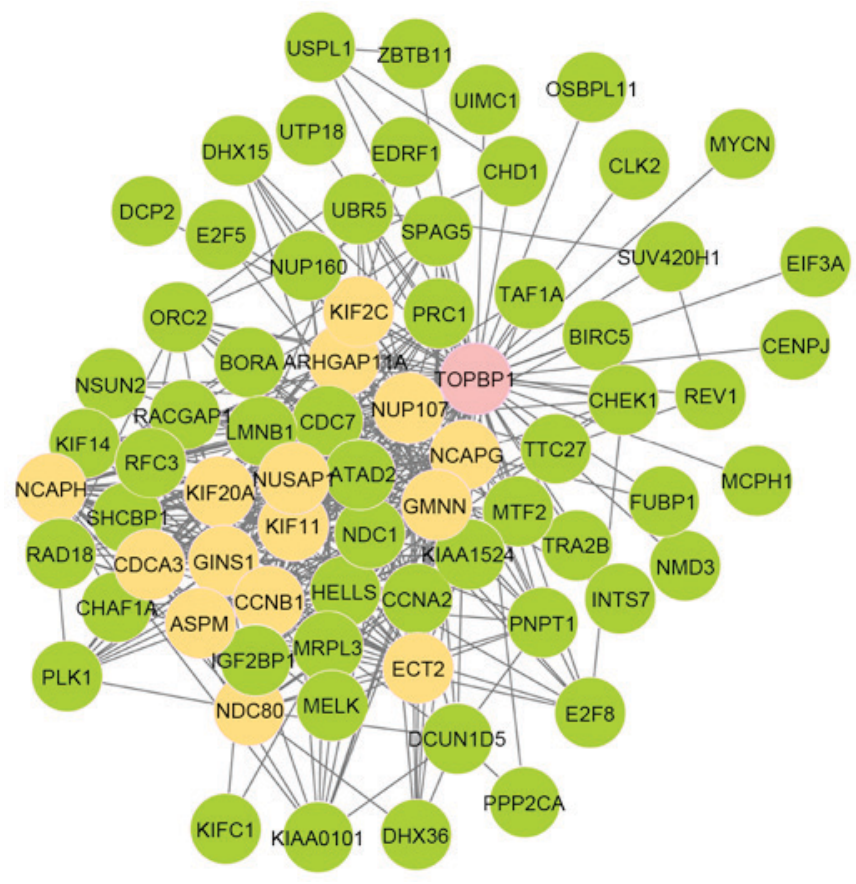

Figure 1. Differential module 11 identified in the differential co-expression network between human hepatocellular carcinoma treated with silymarin and control samples, which included 73 nodes and 420 edges. Pink node, initial seed gene; yellow nodes, seed genes; green nodes, genes interacted with seed genes.

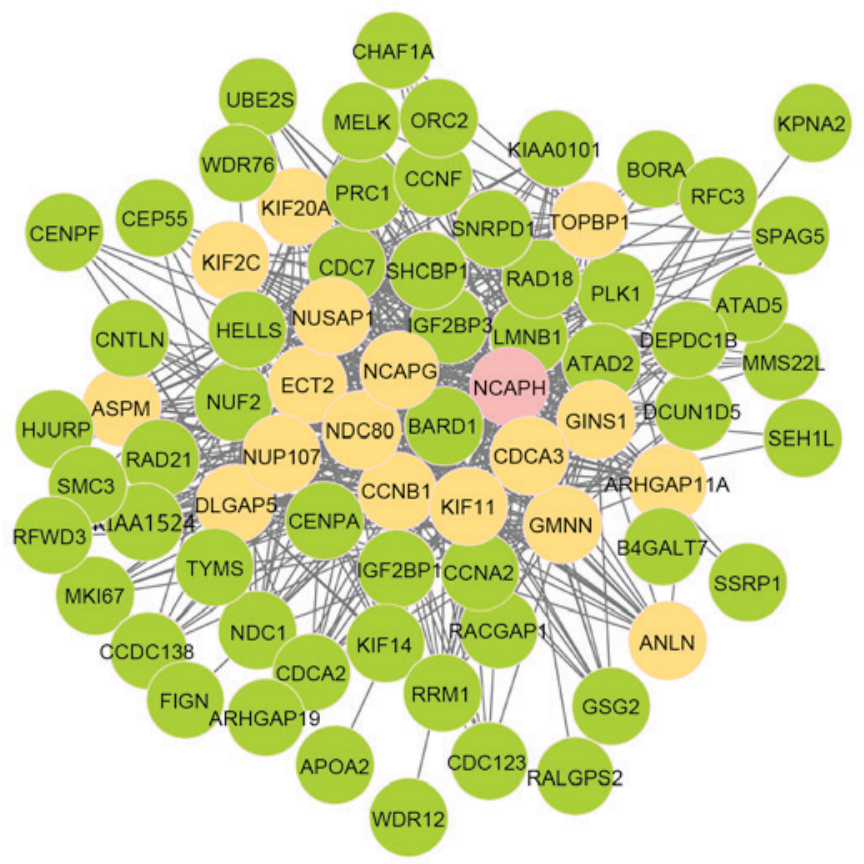

Figure 2. Differential module 5 identified in the differential co-expression network between human hepatocellular carcinoma treated with silymarin and control samples, which included 74 nodes and 553 edges. Pink node, initial seed gene; yellow nodes, seed genes; green nodes, genes interacted with seed genes.

formation of the mitotic spindle in fumonisin B1-induced hepatotoxicity (35). Therefore, it was speculated that the anti-HCC effects of SM may downregulate NUSAP1 to further induce cell-cycle arrest.

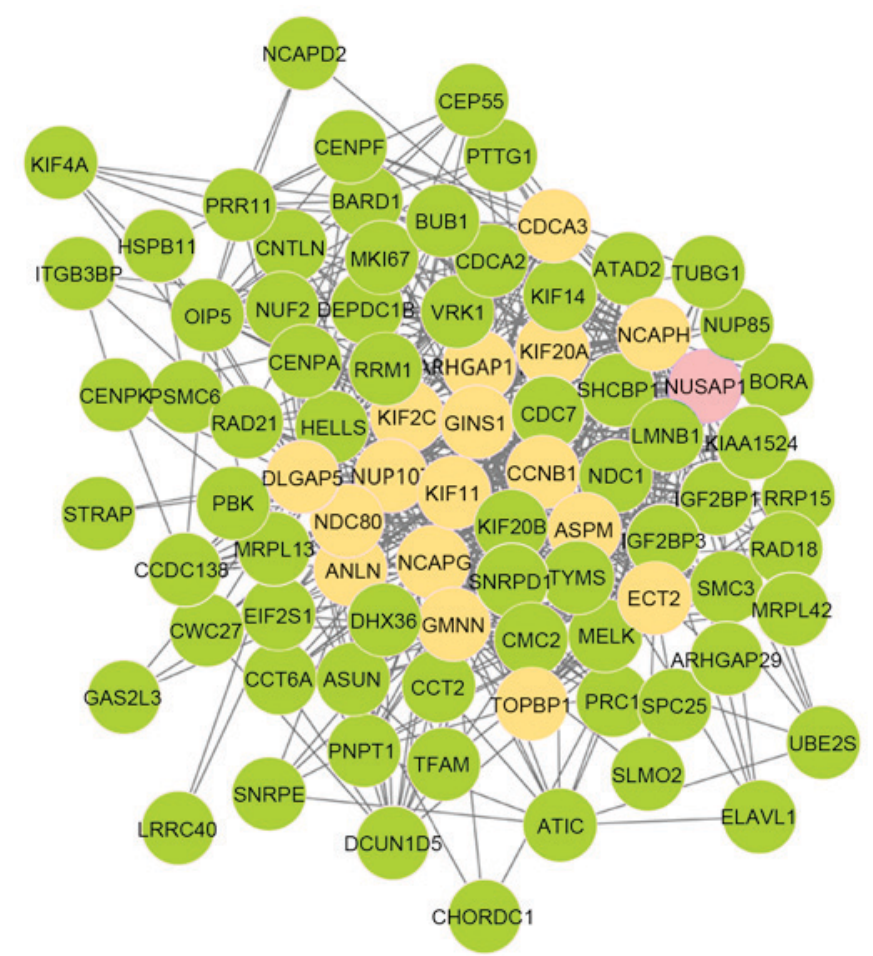

Figure 3. Differential module 6 identified in the differential co-expression network between human hepatocellular carcinoma treated with silymarin and control samples, which included 86 nodes and 634 edges Pink node, initial seed gene; yellow nodes, seed genes; green nodes, genes interacted with seed genes.

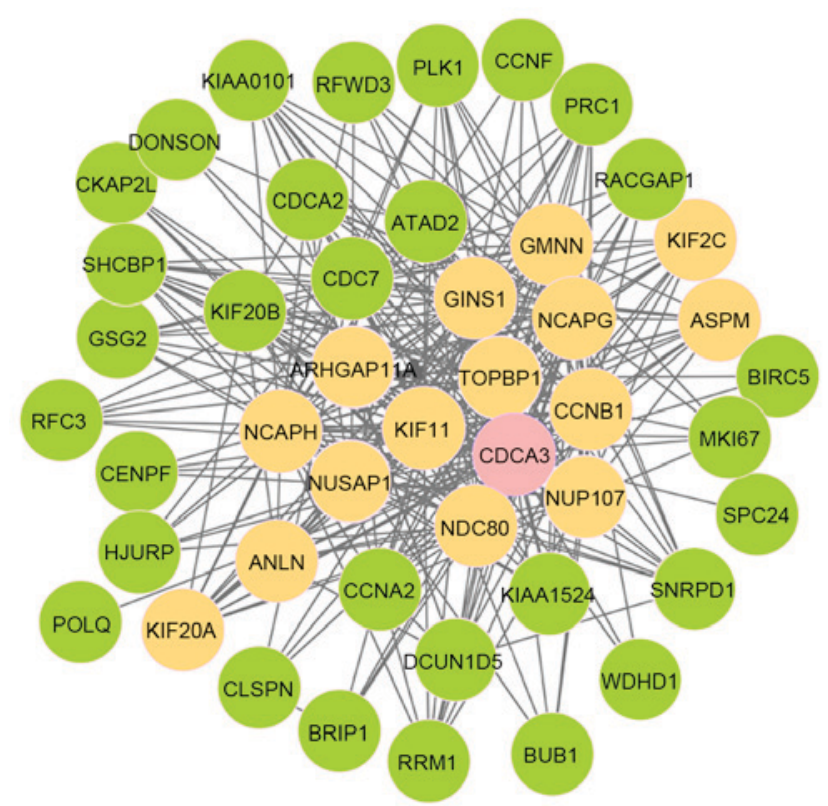

Figure 4. Differential module 12 identified in the differential co-expression network between human hepatocellular carcinoma treated with silymarin and control samples, which included 46 nodes and 315 edges. Pink node, initial seed gene; yellow nodes, seed genes; green nodes, genes interacted with seed genes.

Cancer is characterized by uncontrolled cellular proliferation caused by the dysregulation of the cell cycle (36). CDCA3, a component of Skp1-cullin-F-box, is a regulator of mitosis (37). Notably, kinesin family member 18B and CDCA3 have been 
Table V. Significant pathways for core modules based on FDR $<0.05$.

\begin{tabular}{|c|c|c|c|}
\hline Module & Category & Term & FDR \\
\hline \multirow[t]{2}{*}{11} & KEGG_PATHWAY & Cell cycle & $1.56 \times 10^{-4}$ \\
\hline & & RNA transport & $4.63 \times 10^{-2}$ \\
\hline 5 & KEGG_PATHWAY & Cell cycle & $4.02 \times 10^{-4}$ \\
\hline \multirow[t]{2}{*}{6} & KEGG_PATHWAY & Cell cycle & $3.95 \times 10^{-2}$ \\
\hline & & RNA transport & $2.66 \times 10^{-2}$ \\
\hline \multirow[t]{2}{*}{12} & KEGG_PATHWAY & Cell cycle & $4.57 \times 10^{-4}$ \\
\hline & & $\begin{array}{l}\text { Progesterone-mediated } \\
\text { oocyte maturation }\end{array}$ & $2.54 \times 10^{-3}$ \\
\hline
\end{tabular}

FDR, false discovery rate; KEGG, Kyoto Encyclopedia of Genes and Genomes.

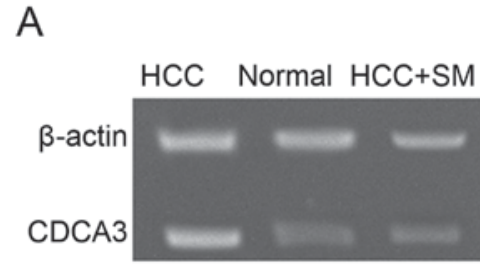

B

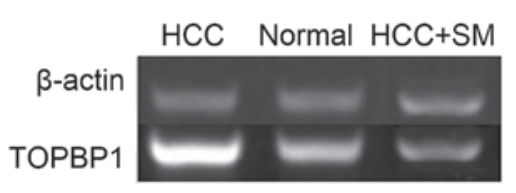

C

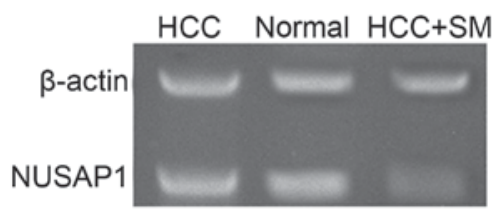

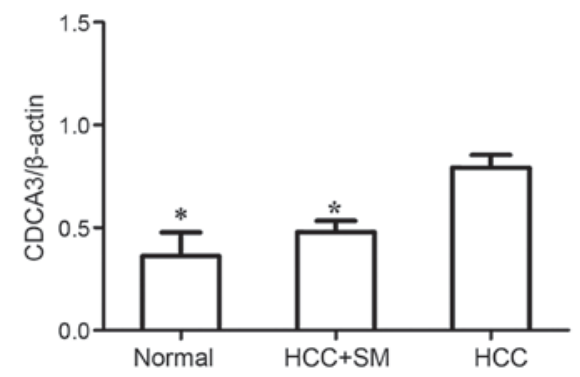
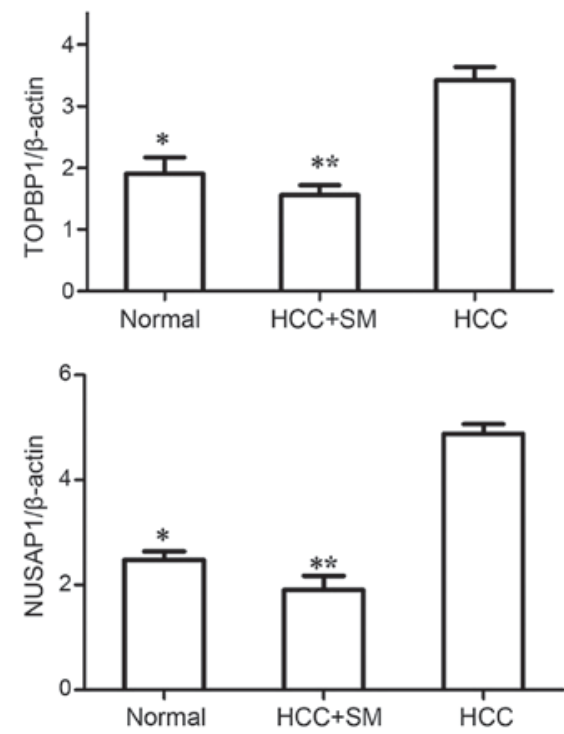

Figure 5. The expression of (A) CDCA3, (B) TOPBP1 and (C) NUSAP1 in normal liver cells, untreated HCC cells and SM-treated HCC cells were investigated using polymerase chain reaction. ${ }^{*} \mathrm{P}<0.05,{ }^{* *} \mathrm{P}<0.001$ vs. HCC group. HCC, hepatocellular carcinoma; $\mathrm{SM}$, silymarin; $C D C A 3$, cell division cycle associated 3 ; TOPBP1, DNA topoisomerase 2-binding protein 1; NUSAP1, nucleolar and spindle associated protein 1.

demonstrated to exert crucial functions in HCC by inducing cell cycle progression (38). Deep et al (39) suggested that SM suppresses cellular proliferation and induces $G_{1}$ and $G_{2}-M$ phase cell cycle arrest in human prostate cancer. Furthermore, another study demonstrated that SM promotes cell cycle arrest in ovarian cancer (40). Hence, the suppression effects of SM on HCC progression may be attributed to the induction of cell cycle arrest, partially via regulating the expression of $C D C A 3$.

On the basis of the PCR results, it was identified that the expression levels of TOPBPI, CDCA3 and NUSAP1 were significantly increased in untreated HCC compared with that in the normal group. Furthermore, following SM treatment in HCC samples, the level of CDCA3, TOPBPI and NUSAP1 was significantly decreased compared with that in the untreated HCC group. These results further demonstrated that patients with HCC may benefit from SM treatment based on the expression of these key genes.

Although several significant gene modules were obtained in the present study, certain limitations must be taken into consideration. First, the sample size was limited. Additionally, the study implemented bioinformatic analysis based on published datasets and the results were validated using the 
samples obtained in the present study; however, no microarray or next-generation sequencing (NGS) analysis was performed on this this data. Therefore, further investigation is required to investigate the molecular mechanisms underlying the anti-HCC effects of SM based on microarray or NGS analysis of the primary data acquired in the present study.

In summary, the results of the present study may provide a theoretical basis of the underlying molecular mechanisms involved in anti-HCC effect of SM treatment. TOPBP1, NUSAP1 and CDCA3 of the core modules may serve important functions in the suppression of HCC by SM. The aforementioned results may assist in the understanding of molecular mechanism of HCC development.

\section{References}

1. Romaguera D, Vergnaud AC, Peeters PH, van Gils CH, Chan DS, Ferrari P, Romieu I, Jenab M, Slimani N, Clavel-Chapelon F, et al: Is concordance with world cancer research fund/American institute for cancer research guidelines for cancer prevention related to subsequent risk of cancer? Results from the EPIC study. Am J Clin Nutr 96: 150-163, 2012.

2. Ferlay J, Soerjomataram I, Dikshit R, Eser S, Mathers C, Rebelo M, Parkin DM, Forman D and Bray F: Cancer incidence and mortality worldwide: sources, methods and major patterns in GLOBOCAN 2012. Int J Cancer 136: E359-E386, 2015.

3. Wang YC, Wei LJ, Liu JT, Li SX and Wang QS: Comparison of cancer incidence between China and the USA. Cancer Biol Med 9: 128-132, 2012.

4. Khan M, Li T, Ahmad Khan MK, Rasul A, Nawaz F, Sun M, Zheng Y and Ma T: Alantolactone induces apoptosis in HepG2 cells through GSH depletion, inhibition of STAT3 activation, and mitochondrial dysfunction. Biomed Res Int 2013: 719858, 2013.

5. Kojima S, Okuno M, Matsushima-Nishiwaki R, Friedman SL and Moriwaki H: Acyclic retinoid in the chemoprevention of hepatocellular carcinoma (Review). Int J Oncol 24: 797-805, 2004.

6. Deep G, Gangar SC, Rajamanickam S, Raina K, Gu M, Agarwal C, Oberlies NH and Agarwal R: Angiopreventive efficacy of pure flavonolignans from milk thistle extract against prostate cancer: Targeting VEGF-VEGFR signaling. PLoS One 7: e34630, 2012.

7. Yurtcu E, Kasapoğlu E and Şahin FI: Protective effects of $\beta$-carotene and silymarin on human lymphocytes. Turk J Biol 36: 47-52, 2012.

8. Ramasamy K and Agarwal R: Multitargeted therapy of cancer by silymarin. Cancer Lett 269: 352-362, 2008.

9. He D, Liu ZP, Honda M, Kaneko S and Chen L: Coexpression network analysis in chronic hepatitis $\mathrm{B}$ and $\mathrm{C}$ hepatic lesions reveals distinct patterns of disease progression to hepatocellular carcinoma. J Mol Cell Biol 4: 140-152, 2012.

10. Liu ZP, Wang Y, Zhang XS and Chen L: Network-based analysis of complex diseases. IET Syst Biol 6: 22-33, 2012.

11. Ma X, Gao L and Tan K: Modeling disease progression using dynamics of pathway connectivity. Bioinformatics 30 2343-2350, 2014

12. Ma S, Calhoun VD, Phlypo R and Adalı T: Dynamic changes of spatial functional network connectivity in healthy individuals and schizophrenia patients using independent vector analysis. Neuroimage 90: 196-206, 2014.

13. Ruan J, Dean AK and Zhang W: A general co-expression network-based approach to gene expression analysis: Comparison and applications. BMC Syst Biol 4: 8, 2010.

14. Mao L, Van Hemert JL, Dash S and Dickerson JA: Arabidopsis gene co-expression network and its functional modules. BMC Bioinformatics 10: 346, 2009.

15. Yang Y, Han L, Yuan Y, Li J, Hei N and Liang H: Gene co-expression network analysis reveals common system-level properties of prognostic genes across cancer types. Nat Commun 5: 3231, 2014.

16. MaX,GaoL,Karamanlidis G,GaoP,LeeCF, Garcia-MenendezL, Tian R and Tan K: Revealing pathway dynamics in heart diseases by analyzing multiple differential networks. PLoS Comput Biol 11: e1004332, 2015.

17. Lovelace ES, Wagoner J, MacDonald J, Bammler T, Bruckner J, Brownell J, Beyer RP, Zink EM, Kim YM, Kyle JE, et al: Silymarin suppresses cellular inflammation by inducing reparative stress signaling. J Nat Prod 78: 1990-2000, 2015.
18. Mar JC, Matigian NA, Quackenbush J and Wells CA: Attract: A method for identifying core pathways that define cellular phenotypes. PLoS One 6: e25445, 2011.

19. Watson-Haigh NS, Kadarmideen HN and Reverter A: PCIT: An R package for weighted gene co-expression networks based on partial correlation and information theory approaches. Bioinformatics 26: 411-413, 2010.

20. Benjamini Y, Drai D, Elmer G, Kafkafi N and Golani I: Controlling the false discovery rate in behavior genetics research. Behav Brain Res 125: 279-284, 2001.

21. Kanehisa M, Araki M, Goto S, Hattori M, Hirakawa M, Itoh M, Katayama T, Kawashima S, Okuda S, Tokimatsu T and Yamanishi Y: KEGG for linking genomes to life and the environment. Nucleic Acids Res 36 (Database issue): D480-D484, 2008.

22. Huang da W, Sherman BT and Lempicki RA: Systematic and integrative analysis of large gene lists using DAVID bioinformatics resources. Nat Protoc 4: 44-57, 2009.

23. Bolstad BM, Irizarry RA, Astrand M and Speed TP: A comparison of normalization methods for high density oligonucleotide array data based on variance and bias. Bioinformatics 19: 185-193, 2003

24. Post-White J,Ladas EJ and Kelly KM: Advances in the use of milk thistle (Silybum marianum). Integr Cancer Ther 6: 104-109, 2007.

25. Bang SW, Ko MJ, Kang S, Kim GS, Kang D, Lee J and Hwang DS: Human TopBP1 localization to the mitotic centrosome mediates mitotic progression. Exp Cell Res 317: 994-1004, 2011.

26. Lee Y, Katyal S, Downing SM, Zhao J, Russell HR and Mckinnon PJ: Neurogenesis requires TopBP1 to prevent catastrophic replicative DNA damage in early progenitors. Nat Neurosci 15: 819-826, 2012.

27. Negrini S, Gorgoulis VG and Halazonetis TD: Genomic instability-an evolving hallmark of cancer. Nat Rev Mol Cell Biol 11: 220-228, 2010.

28. Hanahan D and Weinberg RA: Hallmarks of cancer: The next generation. Cell 144: 646-674, 2011.

29. Katiyar SK, Mantena SK and Meeran SM: Silymarin protects epidermal keratinocytes from ultraviolet radiation-induced apoptosis and DNA damage by nucleotide excision repair mechanism. PLoS One 6: e21410, 2011.

30. Iyer J, Moghe S, Furukawa M and Tsai MY: What's Nu (SAP) in mitosis and cancer? Cell Signal 23: 991-998, 2011.

31. Lu J, Tan M, Huang W C, Li P, Guo H, Tseng LM, Su XH, Yang WT, Treekitkarnmongkol W, Andreeff M, et al: Mitotic deregulation by survivin in ErbB2-overexpressing breast cancer cells contributes to Taxol resistance. Clin Cancer Res 15: 1326-1334, 2009.

32. Mita AC, Mita MM, Nawrocki ST and Giles FJ: Survivin: Key regulator of mitosis and apoptosis and novel target for cancer therapeutics. Clin Cancer Res 14: 5000-5005, 2008.

33. Chen DT, Nasir A, Culhane A, Venkataramu C, Fulp W, Rubio R, Wang T, Agrawal D, McCarthy SM, Gruidl M, et al: Proliferative genes dominate malignancy-risk gene signature in histologically-normal breast tissue. Breast Cancer Res Treat 119: 335-346, 2010.

34. Satow R, Shitashige M, Kanai Y, Takeshita F, Ojima H, Jigami T, Honda K, Kosuge T, Ochiya T, Hirohashi S and Yamada T: Combined functional genome survey of therapeutic targets for hepatocellular carcinoma. Clin Cancer Res 16: 2518-2528, 2010.

35. Sozmen M, Devrim AK, Tunca R, Bayezit M, Dag S and Essiz D: Protective effects of silymarin on fumonisin $\mathrm{B}_{1}$-induced hepatotoxicity in mice. J Vet Sci 15: 51-60, 2014.

36. Urrego D, Tomczak AP, Zahed F, Stuhmer W and Pardo LA: Potassium channels in cell cycle and cell proliferation. Philos Trans R Soc Lond B Biol Sci 369: 20130094, 2014.

37. Smith A, Simanski S, Fallahi M and Ayad NG: Redundant ubiquitin ligase activities regulate weel degradation and mitotic entry. Cell Cycle 6: 2795-2799, 2007.

38. Itzel T, Scholz P, Maass T, et al: Translating bioinformatics in oncology: Guilt-by-profiling analysis and identification of KIF18B and CDCA3 as novel driver genes in carcinogenesis. Bioinformatics 31: 216-224, 2015.

39. Deep G, Singh R, Agarwal C, Kroll D and Agarwal R: Silymarin and silibinin cause G1 and G2-M cell cycle arrest via distinct circuitries in human prostate cancer PC 3 cells: A comparison of flavanone silibinin with flavanolignan mixture silymarin. Oncogene 25: 1053-1069, 2006.

40. Fan L, Ma Y, Liu Y, Zheng D and Huang G: Silymarin induces cell cycle arrest and apoptosis in ovarian cancer cells. Eur J Pharmacol 743: 79-88, 2014.

This work is licensed under a Creative Commons Attribution-NonCommercial-NoDerivatives 4.0 International (CC BY-NC-ND 4.0) License. 